\title{
BRACHYRACHISINA: ISOFLAVONA INÉDITA DE Swartzia (LEGUMINOSAE)
}

\author{
Cesar Sáenz SANCHEZ', Arnaldo F. Imbiriba da ROCHA', M. L. Belém \\ PINHEIRO', Carlos H. S. ANDRADE', Francisco José Queiroz MONTE
}

RESUMO - Dos extratos benzênico e clorofórmico da madeira de Swartzia brachyrachis Harms var: brachyrachis foram isolados, por métodos cromatográficos, uma isoflavona inédita (7,4'-diidroxi -5,3',5'-trimetoxi-6-metilisoflavona), denominada brachyrachisina, e um glicosídio (3-O- $\beta$-Dglicopiranosilsitosterol). As estruturas foram elucidadas através de análises espectroscópicas.

Palavras-chave: Swarlzia; 3-O- $\beta$-D-glicopiranosilsitosterol; brachyrachisina; 7,4'-diidroxi5,3',5'-trimetoxi-6-metilisoflavona.

\section{Brachyrachisine: a New Isoflavone from Swartzia (Leguminosae)}

ABSTRACT - From the wood benzene and chloroform extracts of Swartzia brachyrachis Harms var.brachyrachis were isolated, by chromatografph methods, an undocumented isoflavone (7,4'-dihydroxy-5,3',5'-trimethoxy-6-methylisoflavone) named brachyrachisine and a glucoside (3-O- $\beta$-D-glucopyranosylsitosterol). The structures were elucidated by spectroscopic analysis.

Key words: Swartzia; 3-O- $\beta$-D-glucopyranosylsitosterol; brachyrachisine; 7,4'-dihydroxy-5,3',5'trimethoxy-6-methylisoflavone.

\section{INTRODUÇÃO}

O gênero Swartzia é constituído de 137 espécies, duas ocorrem na África e as demais na América. A Amazônia é o seu centro de dispersão, espalhando-se ao longo da bacia do Rio Amazonas, desde sua nascente no Peru até o litoral brasileiro, atingindo o sul da Venezuela e da Guiana e a parte ocidental da Colombia. A espécie Swartzia brachyrachis Harms varbrachyrachis distribui-se nos estados do Amazonas, Pará e Amapá (Cowan,1967). Vários representantes do gênero são utilizados na etnomedicina, especialmente como analgésicos e no tratamento de afecções hepáticas (Schultes,1979). Dada a marcante presença de isoflavonóides entre os constituintes químicos do táxon, particularmente pterocarpanos, e as atividades biológicas relatadas para as cabenegrinas (Nakagawa, 1982), seu estudo tem merecido atenção pela possibilidade de apresentar substâncias com ação contra veneno de cobra.

O extrato benzênico levou ao isolamento da isoflavona inédita 7,4'diidroxi-5, 3', 5' -trimetoxi-6metilisoflavona 1 , denominada brachyrachisina, e do glicosídio 3-O$\beta$-D-glicopiranosilsitosterol 2, anteriormente reportado na literatura (Macari, 1990) e também isolado do extrato clorofórmico.

\section{RESULTADOS E DISCUSSÃO}

Os extratos orgânicos foram preparados a partir de material botânico seco e pulverizado, através de maceração sucessiva com benzeno e clorofórmio, à temperatura ambiente.

1 Departamento de Química, Instituto de Ciências Exatas, Universidade do Amazonas- Av. General Rodrigo Otávio Jordão Ramos, 3000- Manaus (AM)-CEP-69 070000

2 Departamento de Quimica Orgânica e Inorgânica, Centro de Ciências - Universidade Federal do Ceará- Campus do Pici- Fortaleza (CE) - CEP 60021970. 
A separação/purificação dos fitoconstituintes foi realizada em colunas cromatográficas de sílica gel. O composto 1 , isolado como um sólido cristalino esbranquiçado, foi inicialmente identificado como um isoflavonóide através de sinais característicos observados nos espectros de $\mathrm{RMN}{ }^{1} \mathrm{H}$, em $\delta_{\mathrm{H}} \quad 7,74$ (s,1H, H-2) (Wong, 1975) e de RMN ${ }^{13} \mathrm{C}$, em $\delta_{\mathrm{C}}$ 153,9 (C-2) (Agrawal \& Bansal, 1989) . O esqueleto isoflavônico foi ratificado pelos espectros nas regiões do UV $\lambda_{\text {max }} n m(\log \varepsilon): 353,9(0,652)$, $290,7(0,168)$ e $224,4(0,729)$ (Mabry,1970) e IV $v_{\max }{ }^{\mathrm{KBr}} \mathrm{cm}^{-1}$ :inter al $1725(\mathrm{C}=\mathrm{O}), 1624,1561,1498$, 1470 (sistema aromático). A presença de três grupos metoxila e dois grupos acetoxila foi revelada, no espectro de RMN ${ }^{1} \mathrm{H}$ do derivado acetilado $1 \mathrm{a}$, pelos sinais em $\delta_{\mathrm{H}} 4,02(\mathrm{~s}, 3 \mathrm{H}), \delta_{\mathrm{H}}$ $3,90(\mathrm{~s}, 6 \mathrm{H})$ e $\delta_{\mathrm{H}} 2,39(\mathrm{~s}, 3 \mathrm{H}), \delta_{\mathrm{H}} 2,34$ $(\mathrm{s}, 3 \mathrm{H})$, respectivamente, e no espectro de massas, pelas sucessivas perdas de aldeído fórmico e ceteno. A ausência de função hidroxila quelada em C-5 e de sistema orto-diidroxilado, para o composto 1 , foi indicada pela não alteração do seu espectro no UV, em presença de $\mathrm{AlCl}_{3}$. O deslocamento da banda II, em presença de $\mathrm{NaOAc}$, sugeriu o posicionamento de uma hidroxila em C-7. Neste ponto, por considerações biogenéticas, foi permitido localizar um grupo metoxila em C-5. Por outro lado, a equivalência dos dois grupos metoxila $\left(\delta_{\mathrm{H}} 3,90, \mathrm{~s}\right.$, $6 \mathrm{H}$ ) indicou a posição C-4' para a outra hidroxila, ficando as alternativas C-2'/C-6' ou C-3'/C-5' para as duas metoxilas simetricamente distribuídas.
$\mathrm{O}$ singleto em $\delta_{\mathrm{H}} 7,26(2 \mathrm{H})$ foi correlacionado a H-2'e H-6' (Mabry, 1970) e assim definido o padrão de substituição do anel B. Esta dedução está apoiada pela presença do fragmento em $\mathrm{m} / \mathrm{z} 358$ (pico base, resultante da perda de 84 daltons, correspondendo a eliminação de duas moléculas de ceteno), registrado no espectro de massas de la. Com base na análise de espectros de $\mathrm{RMN}$ ' $\mathrm{H}$ de sistemas análogos registrados na literatura (Mabry, 1970), o singleto em $\delta_{\mathrm{H}} 2,33(3 \mathrm{H})$ foi atribuido a um grupo metila localizado em C-6. Finalmente, o terceiro singleto de hidrogênio aromático, observado em $\delta_{\mathrm{H}} 6,64(1 \mathrm{H})$ foi correlacionado ao H-8 (Mabry, 1970), sendo o sinal em campo relativamente alto, justificado pela localização deste hidrogênio em um sistema 5,7,9- trioxigenado. Estes dados definiram a estrutura 1 para a isoflavona inédita, denominada brachyrachisina. O glicosídio, isolado de ambos os extratos (benzênico e clorofórmico) e identificado sob forma de acetato, foi caracterizado como 3-O- $\beta$-Dglicopiranosilsitosterol por comparação de seus dados espectrométricos, principalmente de $\mathrm{RMN}{ }^{13} \mathrm{C}$, com os da literatura (Macari,1990).

\section{MATERIAL E MÉTODOS}

Geral - Os espectros no UV foram realizados em espectrômetro Perkin Elmer, Lambda 11, usando metanol como solvente. Os espectros no IV foram registrados em aparelho Perkin Elmer, modelo Spectrum 2000 /FT-IR, utilizando pastilhas de $\mathrm{KBr}$. Os espectros de massa foram obtidos em 
aparelho Finningan 3.200,operando a $70 \mathrm{eV}$. Os espectros de $\mathrm{RMN}{ }^{1} \mathrm{H}$ e $\mathrm{RMN}{ }^{13} \mathrm{C}$ foram registrados em um espectrômetro Varian, modelo VXR360 , respectivamente a 200 e 50,3 $\mathrm{MHz}$, usando $\mathrm{CDCl}_{3}$ como solvente e TMS como padrão.

Planta - O material botânico de S.brachyrachis Harms varbrachyrachis foi coletado em outubro de 1994, na reserva florestal Ducke/Instituto Nacional de Pesquisas da Amazônia (INPA), Km 27 da estrada ManausItacoatiara e está registrado no herbário do INPA-Manaus, sob o $\mathrm{N}^{\circ} 15.455$.

Isolamento e purificação dos constituintes - $2440 \mathrm{~g}$ de madeira da planta seca e moída foram macerados, sucessivamente, com benzeno e clorofórmio. O extrato benzênico $(3,2 \mathrm{~g})$ foi cromatografado em uma coluna de sílica gel, utilizando como eluentes, hexano, acetato de etila e metanol. A fração acetato de etila forneceu dois sólidos, rotulados como Fr-13 e Fr-17. Após lavagem com hexano, $\mathrm{Fr}-13$ foi dissolvido em clorofórmio e deixado na geladeira durante uma noite. $\mathrm{O}$ precipitado gerado foi centrifugado e lavado com benzeno, resultando o sólido cristalino esbranquiçado 1. Uma porção de 1 foi acetilada com piridina/ anidrido acético, sendo obtido la. A Fr.17 foi recristalizada em metanol, fornecendo o sólido 2, que acetilado gerou 2a. O extrato clorofórmico foi submetido a fracionamento cromatográfico semelhante ao anteriormente citado. Das frações eluidas com acetato de etila obteve-se um sólido que recristalizado em metanol forneceu 2, também isolado do extrato benzênico.

Dados espectrométricos: 7,4'- diidroxi-5, 3', 5' -trimetoxi-6metilisoflavona (1)- UV $\lambda_{\text {max }} \mathrm{MeOH}$ nm (log \&): 353,9 (0,652), 290,7 $(0,168)$ e $224,4(0,729)$. IV $v_{\max }{ }^{\mathrm{KBr}} \mathrm{cm}^{-1}:$ inter al 1725, 1624, 1561, $14981470 \mathrm{~cm}^{-1} .7$, 4'-diacetoxi-5,3',5'-trimetoxi-6metilisoflavona (1a)- EM, m/z (\%): $442\left[\mathrm{M}^{+}\right]$(39), 412 (15), 400 (25), 370 (13) 358 (100), 343 (28), 328 (48) 313 (23). RMN'H: 7,74 (s, 1H, H-2), 7,26 (s, 2H, H-2'e H-6'), 6,64 (s, 1H, H-8), $4,02\left(\mathrm{~s}, 3 \mathrm{H}, \mathrm{CH}_{3} \mathrm{O}\right), 3,90(\mathrm{~s}, 6 \mathrm{H}$, $\left.2 \mathrm{CH}_{3} \mathrm{O}\right), 2,39\left(\mathrm{~s}, 3 \mathrm{H}, \mathrm{CH}_{3} \mathrm{CO}\right), 2,34$ (s,3H, $\left.\mathrm{CH}_{3} \mathrm{CO}\right), 2,33\left(\mathrm{~s}, \mathrm{CH}_{3}\right) \cdot \mathrm{RMN}^{13} \mathrm{C}-$ $169,2,\left(\mathrm{CH}_{3} \underline{\mathrm{C}}=\mathrm{O}\right), 168,5\left(\mathrm{CH}_{3} \underline{\mathrm{C}}=\mathrm{O}\right)$, $155,0,153,9$ (C-2), 151,0, 136,4, 115,0, $114,8,97,1,96,2$ (aromáticos), 62,5 $\left(\mathrm{CH}_{3} \mathrm{O}\right), 56,6\left(\mathrm{CH}_{3} \mathrm{O}\right), 56,4\left(\mathrm{CH}_{3} \mathrm{O}\right), 29,7$ $\left(\mathrm{CH}_{3} \mathrm{CO}\right), 20,6\left(\mathrm{CH}_{3}\right)$.

\section{AGRADECIMENTOS}

Os autores agradecem à CAPES pela bolsa de mestrado concedida a Cesar Sáenz Sanchez.

\section{Bibliografia Citada}

Agrawal, P. K.; Bansal, M.C. 1989. Carbon-13 NMR of Flavonoids In: Agrawal, P.K. (ed). Studies in Organic Chemistry. vol.39, Ed. Elseier Science Publishers, Amsterdam, p 192.

Cowan, R.S. 1967. Swartzia (Leguminosae, Caesalpinioideae, Swartzieae). Flora Neotrópica, Monograph No.l. Hafner Publishing Company. New York, London. 228p,

Mabry, T. J.; Markham, K.R.; Thomas, M. B. 1970. The Systematic Identification of Flavonoids, Springer-Verlag, New York., Heidelberg, Berlin. 354p.

Macari, P.A.; Emereciano, V.de.P.; Ferreira, Z.M.G.S. 1990. Identificação dos triterpenos de Micconia Albicans Triana, através da análise por microcomputador. Quimica Nova 13 (4): 260-262.

Nakagawa M.; Nakanishi, K. 1982. Structures 
of cabenegrines A-I and A-II. Potent antisnake venoms. Tetrahedron Letters 10 (38): 3855-3858.

Schultes, R.E. 1979.De Plantis Toxicariis e Mundo Novo tropicale commentationes, XX Medicinal and toxic uses of Swartzia in the northwest Amazon. Journal of Ethnopharmacology, 1(1): 79-87.
Wong, E. 1975. In The Flavonoids, Eds. Harborny, J.B., Mabry, T.J., Mabry, H., Academic Press, New York. p.746.<smiles>[R]c1cc2occ(-c3cc(OC)c([R])c(OC)c3)c(=O)c2c(OC)c1C</smiles>

$1, \mathrm{R}=\mathrm{OH}$

1a, $\mathrm{R}=\mathrm{OCOCH}_{3}$

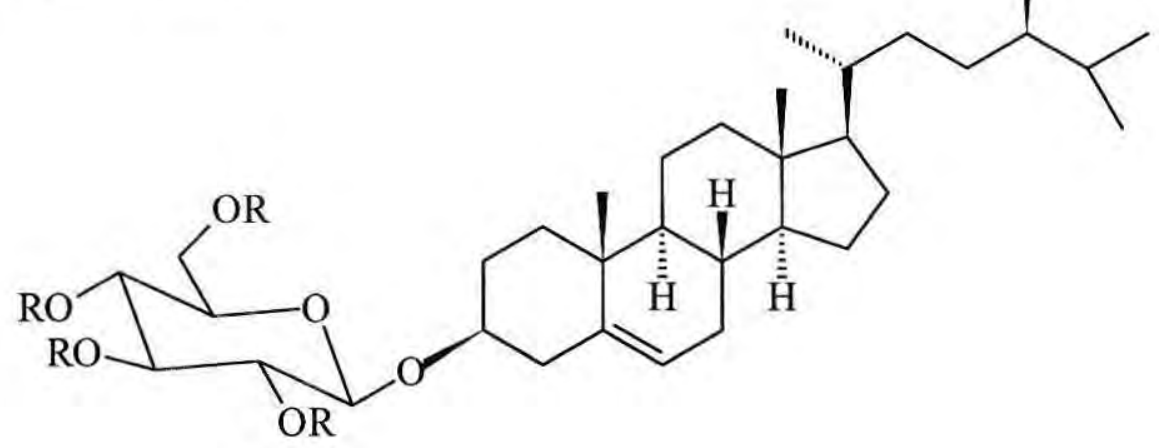

2, $\mathrm{R}=\mathrm{H}$

$2 \mathrm{a}, \mathrm{R}=\mathrm{COCH}_{3}$ 